\title{
Direct-current-dependent shift of theta-burst-induced plasticity in the human motor cortex
}

\author{
Alkomiet Hasan • Masashi Hamada \\ Michael A. Nitsche $\cdot$ Diane Ruge $\cdot$ Joseph M. Galea $\cdot$ \\ Thomas Wobrock $\cdot$ John C. Rothwell
}

Received: 25 August 2011 / Accepted: 23 November 2011 / Published online: 6 December 2011

(C) The Author(s) 2011. This article is published with open access at Springerlink.com

\begin{abstract}
Animal studies using polarising currents have shown that induction of synaptic long-term potentiation (LTP) and long-term depression (LTD) by bursts of patterned stimulation is affected by the membrane potential of the postsynaptic neurone. The aim of the present experiments was to test whether it is possible to observe similar phenomena in humans with the aim of improving present protocols of inducing synaptic plasticity for therapeutic purposes. We tested whether the LTP/LTD-like after effects of transcranial theta-burst stimulation (TBS) of human motor cortex, an analogue of patterned electrical stimulation in animals, were affected by simultaneous transcranial direct-current stimulation (tDCS), a non-invasive method of polarising cortical neurones in humans. Nine healthy volunteers were investigated in a single-blind, balanced cross-over study; continuous TBS (cTBS) was used to introduce LTD-like after effects, whereas intermittent TBS (iTBS) produced LTP-like effects. Each pattern was coupled with concurrent application of tDCS $(<200 \mathrm{~s}$, anodal, cathodal, sham). Cathodal tDCS increased the response to iTBS and abolished the effects of cTBS. Anodal tDCS
\end{abstract}

A. Hasan $(\varangle) \cdot$ M. Hamada · D. Ruge · J. M. Galea · J. C. Rothwell Sobell Department of Motor Neuroscience and Movement Disorders, Institute of Neurology, University College London, Room 3.10, 33 Queen Square, Box 146, London WC1N 3BG, UK e-mail: ahasan@gwdg.de

A. Hasan · T. Wobrock

Department of Psychiatry and Psychotherapy, University of Goettingen, Von-Siebold-Street 5,

37075 Goettingen, Germany

M. A. Nitsche

Department of Clinical Neurophysiology,

University of Goettingen, Robert-Koch-Street 20,

37075 Goettingen, Germany changed the effects of cTBS towards facilitation, but had no impact on iTBS. Cortical motor thresholds and intracortical inhibitory/facilitatory networks were not altered by any of the stimulation protocols. We conclude that the after effects of TBS can be modulated by concurrent tDCS. We hypothesise that tDCS changes the membrane potential of the apical dendrites of cortical pyramidal neurones and that this changes the response to patterned synaptic input evoked by TBS. The data show that it may be possible to enhance LTP-like plasticity after TBS in the human cortex.

Keywords Motor cortex plasticity $\cdot$ Long-term potentiation $\cdot$ Long-term depression $\cdot$ Plasticity regulation

\section{Introduction}

In the motor cortex, transcranial magnetic stimulation (rTMS) can increase or decrease the response to a standard TMS pulse for $30 \mathrm{~min}$ or more after stimulation. Since these effects are abolished by drugs that block NMDA receptors (NMDAR), they are thought to involve cortical long-term potentiation (LTP)- or long-term depression (LTD)-like synaptic plasticity (Ziemann et al. 2008; Ziemann and Siebner 2008).

A common type of rTMS that was introduced in 2005 is theta-burst stimulation (TBS) (Huang et al. 2005). TBS can produce significant and long-lasting LTP- and LTD-like effects within very short stimulation periods. If TBS is applied in an intermittent pattern (iTBS) to the human motor cortex, it enhances the amplitudes of motor-evoked potentials (MEP), whereas the application of a continuous train of stimuli (cTBS) suppresses MEPs (Huang et al. 2005). Huang et al. $(2005,2010)$ speculated that all patterns of TBS produce a mixture of excitatory and inhibitory after 
effects and that the final effect of any particular paradigm depends on the balance between them. To explain this, they suggested that glutamate release during TBS activates NMDAR of the postsynaptic neurone which in turn depolarises the neuronal membrane and allows $\mathrm{Ca}^{2+}$ to enter the cell. Following one version of the $\mathrm{Ca}^{2+}$-hypothesis of synaptic plasticity (Artola and Singer 1993), the authors proposed that LTD-like effects are related to the total amount of $\mathrm{Ca}^{2+}$-entry whereas LTP was related to the rate of $\mathrm{Ca}^{2+}$-entry.

Transcranial direct-current stimulation (tDCS) is thought to polarise cortical neurones and alter their discharge rates by biasing their membrane potentials towards a more depolarised or hyperpolarised state. If tDCS is applied for longer than 5 min, NMDA receptor and $\mathrm{Ca}^{2+}$-dependent long-lasting after effects on cortical excitability are induced (Nitsche et al. 2008; Nitsche and Paulus 2001). Short periods of tDCS ( $<2 \mathrm{~min}$ ) have no lasting effects on cortical excitability. There have been a number of studies on the interaction between sequential application of long lasting tDCS ( $>5 \mathrm{~min}$ ) and rTMS (Ridding and Ziemann 2010); however, there are no studies of the effects of concurrent tDCS and rTMS/TBS, apart from one experiment which combined paired associative stimulation (PAS) and tDCS (Nitsche et al. 2007).

We aimed to explore the impact of short-lasting, concurrent tDCS on cTBS and iTBS protocols. Experiments on slice preparations of adult rat visual cortex have shown that the induction of LTP and LTD by burst stimulation depends on the membrane potential of the postsynaptic neurons and that the effects of burst stimulation can be modified by external hyper/depolarisation (Artola et al. 1990). It was postulated that biasing the membrane potential changed the rate or amount of $\mathrm{Ca}^{2+}$-entry into the postsynaptic neurone. Thus, we hypothesised that in humans, concurrent tDCS will modify or bias the responses to TBS according to the polarity of DC stimulation in a similar fashion.

\section{Materials and methods}

Nine healthy subjects (two women, seven men, mean age $=30.3 \pm 1.5$ years) participated in this study after giving informed consent. All subjects were right-handed according to the Edinburgh handedness inventory (Oldfield 1971), and none of the subjects had a history of neurological or mental illness or had metallic cerebral implants. No subject had a history of alcohol or drug abuse and nobody was taking any neuroactive medication. The study protocol, which is in accordance with the Declaration of Helsinki, was approved by the Ethics Committee of the University College London.
Theta-burst stimulation

TBS was applied according to previously published protocols (Huang et al. 2005). In short, each burst consists of three stimuli with a repetition rate of $50 \mathrm{~Hz}$, and the bursts were repeated with a frequency of $5 \mathrm{~Hz}$. We applied a continuous train of 20 bursts (cTBS, 600 pulses, LTD-like plasticity) and an intermittent pattern of 20 trains of 10 bursts of $2 \mathrm{~s}$ duration with a break duration of $8 \mathrm{~s}$ between each train (iTBS, 600 pulses, LTP-like plasticity). The conditioning intensity was set at $80 \%$ of active motor threshold (AMT) elicited by a biphasic stimulator. As we performed TBS through the tDCS electrode (thickness approximately $5 \mathrm{~mm}$ ), we had to increase the stimulator output to account for the distance between TMS coil and scalp. Therefore, all biphasic thresholds were measured through the electrodes in all experimental conditions.

\section{Transcranial direct-current stimulation}

tDCS was applied with an intensity of $1 \mathrm{~mA}$ using a commercially available DC stimulator (Eldith-Electro-Diagnostic \& Therapeutic Systems GmbH, Germany, distributed by Magstim Co., Whitland, Dyfeld, UK) through salinesoaked surface sponge electrodes $\left(35 \mathrm{~cm}^{2}\right)$. In each experimental session, the motor cortex electrode (anode or cathode) was placed over the hot spot as identified by TMS and the other electrode was placed above the right orbit (Nitsche and Paulus 2000).

Transcranial magnetic stimulation

During all experiments, subjects were placed in a comfortable armchair with head and arms at rest. We recorded surface electromyography (EMG) from the right first-dorsalinterosseous muscle (FDI) via $\mathrm{Ag} / \mathrm{AgCl}$ electrodes in a belly tendon montage. Raw signals were amplified (Digitimer 360, Digitimer Ltd., Welwyn Garden City, Herts, UK), band-pass filtered $(10-3 \mathrm{kHz})$ and digitalised using a 1401 data acquisition interface (Cambridge Electronic Design Ltd., Cambridge UK) controlled by Signal Software (Cambridge Electronic design). All data were stored on a computer and offline analysed using Signal Software. We controlled for complete relaxation of the target muscle through visual feedback of EMG activity on a computer screen.

This procedure was described previously by our study group (Huang et al. 2005; Talelli et al. 2007). TMS was performed using two identical standard $70 \mathrm{~mm}$ figure of eight coils (Magstim Co., Whitland, Dyfeld, UK) connected either to a monophasic Magstim $200^{2}$-Bistim² system (Magstim Co., Whitland, Dyfeld, UK, single-pulse TMS and corticospinal excitability measurement) or to a 
biphasic Magstim Rapid²-system (Magstim Co., Whitland, Dyfeld, UK, TBS-intervention).

During the experiments, the coils were placed tangentially to the skull above the left primary motor cortex (M1) with the handle pointing away with a $45^{\circ}$ angle from the midsagittal line. This orientation leads to a posterior-anterior directed current, which is oriented perpendicular to the central sulcus and which is optimal to result in a predominantly transsynaptic activation of motor cortex neurons (Di Lazzaro et al. 1998). The optimal stimulation point ("hot spot") was defined as the position where single-pulse TMS induced consistently the largest motor-evoked potentials (MEP). To ensure a constant coil position during the experiment, the hot spot was marked with a skin marker.

\section{Monitoring of excitability changes}

All measures were performed with a monophasic transcranial magnetic stimulator. Before and 3 min after each stimulation procedure, the resting motor threshold (RMT) and the active motor threshold (AMT) were obtained according to standard publications (Rothwell et al. 1999; Ziemann et al. 1996a). To determine corticospinal excitability (MEP size) before and after each stimulation procedure, singlepulse TMS was performed at an intensity to evoke MEPs of about $1 \mathrm{mV}$ ( $\mathrm{S} 1 \mathrm{mV}$, peak to peak, $0.7-1.3 \mathrm{mV}$ ) over the left motor cortical representation of the right FDI. We measured 40 MEPs at baseline and 20 MEPs at different timepoints $(0,5,10,15,20,25,30 \mathrm{~min})$ after the stimulation.

Short-latency intracortical inhibition (SICI) and intracortical facilitation (ICF) were recorded with a standardised paired-pulse protocol (conditioning stimulus: 80\% RMT, test stimulus: $\mathrm{S} 1 \mathrm{mV}$, interstimulus intervals (ISI): 2, 3, 7, 10 and $12 \mathrm{~ms}$ Kujirai et al. 1993). The test pulse was applied 16 times, and all paired-pulses were applied 8 times in a randomised order at $0.25 \mathrm{~Hz}$. SICI and ICF were recorded at baseline and 5 min after stimulation. The intensity of the test pulse was not adjusted since percent SICI/ ICF is known to be unaffected by test pulse amplitude over the range of MEP sizes used in the present experiments (Ridding et al. 1995). SICI/ICF were evaluated before and 5 min after intervention.

\section{Experimental design}

To assess the effect of the simultaneous application of tDCS and TBS, all nine subjects were tested on six different days, resulting in 56 experimental sessions. The study was designed as a single-blind and balanced complete crossover study in a repeated measurement design. Each subject received the following experimental conditions: cathodaltDCS + iTBS, anodal-tDCS + iTBS, sham-tDCS + iTBS, cathodal-tDCS + cTBS, anodal-tDCS + cTBS and sham-
tDCS + cTBS in different sessions separated by at least 4 days from each other.

As tDCS and TBS were performed simultaneously, the TMS coil was placed above the tDCS electrode and the TBS stimulation had to be performed through the tDCS electrode (coil-scalp-distance: approximately $5 \mathrm{~mm}$ ). In all iTBS conditions, the duration of the TBS train was $190 \mathrm{~s}$ and the duration of tDCS was $180 \mathrm{~s}$ (plus $10 \mathrm{~s}$ each of fade in and fade out). In all cTBS conditions, the TBS train lasted for $40 \mathrm{~s}$ and the overlapping tDCS duration was $30 \mathrm{~s}$ (plus $10 \mathrm{~s}$ each of fade in and fade out). For tDCS, this short stimulation period $(<200 \mathrm{~s})$ is known not to produce any after effects, although it changes motor cortical excitability during the stimulation (Nitsche et al. 2008; Nitsche and Paulus 2000). The usual form of sham tDCS applies a ramp of stimulation at the start and end of the stimulation period, with a total duration of $1 \mathrm{~min}$ or so, depending on the exact parameters used. Our stimulation period for cTBS was shorter than this so that in this study our sham tDCS consisted of placing the stimulating pads on the scalp without passing any current between them. Since tDCS was always accompanied by concurrent TBS, participants were unable to distinguish this from real tDCS.

\section{Statistical methods}

SPSS 18 for Windows was used for all analysis, and the level of significance was defined as $\alpha=0.05$. Normal distribution of the data was confirmed using the KolmogrovSmirnov test $(P>0.05)$ for all dependent variables.

To test the timecourse of plasticity changes, a RMANOVA $(8 \times 6)$ with the factors "timecourse" (baseline, 0 min, $5 \mathrm{~min}, 10 \mathrm{~min}, 15 \mathrm{~min}, 20 \mathrm{~min}, 25 \mathrm{~min}, 30 \mathrm{~min}$ ) and "condition" (cathodal-tDCS + iTBS, anodal-tDCS + iTBS, sham-tDCS + iTBS, cathodal-tDCS + cTBS, anodal-tDCS + cTBS, sham-tDCS + cTBS) was performed. Since there was no "timecourse $\mathrm{x}$ condition" interaction on the data points post-TBS in the overall ANOVA, we averaged all timepoints to give a mean post-TBS excitability measure for all consecutive statistical analyses.

Separate repeated measures ANOVAs (RM-ANOVA, $2 \times 6$ ) were calculated with the dependent variables MEP size, thresholds (RMT or AMT) and with the within subject factors "time" (pre and post) and "condition" (cathodal-tDCS + iTBS, anodal-tDCS + iTBS, sham-tDCS + iTBS, cathodal-tDCS + cTBS, anodal-tDCS + cTBS, sham-tDCS + cTBS). If appropriate (significant interactions in the linear model), student's $t$ tests (paired, two-tailed) were performed to determine more specifically whether MEP amplitudes differed before and after stimulation within and between conditions. $T$ Tests were not adjusted for multiple comparison in cases of significant interaction in the linear model. 
Pearson correlation analyses (two-tailed) were performed within iTBS and cTBS conditions to investigate correlations between the plasticity responses, expressed as the post/pre-ratio of the MEP size, itself.

In the linear models, sphericity was tested with the Mauchly's test and if necessary (Mauchly's test $<0.05$ ), the Greenhouse-Geisser correction was used. All data are presented as mean \pm standard error of the mean (SEM), unless otherwise indicated.

\section{Results and statistical analysis}

\section{Modification of cortical plasticity}

Figure 1 illustrates the effects of the 6 conditioning protocols on MEP amplitude. Figure 1a, b shows the mean data at all timepoints from pre-TBS to $30 \mathrm{~min}$ post-TBS (timecourse). Figure 1c, d plot the mean pre- and post-TBS MEP amplitudes of all 6 TBS/tDCS combinations; Fig. 1c depicts data from iTBS interventions, whereas Fig. 1d illustrates data from cTBS interventions. Figure 1e provides a visual summary of the results in terms of the percentage change of MEP after application of TBS. As reported in the statistical analysis below, iTBS alone weakly facilitated MEPs, an effect that was strengthened by simultaneous cathodal tDCS, whereas it was unaffected by simultaneous anodal tDCS. In contrast, cTBS alone suppressed MEPs, but in the presence of simultaneous cathodal or anodal tDCS, this was abolished (turned into facilitation).

Since there was no significant "timecourse x condition" interaction in the first RM-ANOVA, we used the mean postTBS MEP amplitude for the main analysis below (see Table 1). The RM-ANOVA with main factors of "condition" (i.e. TBS/TDCS paradigm) and "time" (pre/post-TBS) revealed significant effects of "condition" $(P=0.030)$, "time" $(P=0.039)$ and a "time" $\times$ "condition" interaction $(P=$ 0.031). Given the significant interaction the remainder of the analysis concentrates on comparing conditions within the cTBS and iTBS subsets (Fig. 2).

Post hoc $t$ tests showed that baseline MEP amplitudes did not differ between conditions (all $P>0.05$ ). However, in the cathodal-tDCS + iTBS condition, MEPs after stimulation were larger than in the sham-tDCS + iTBS
Fig. 1 Effects of the different stimulation protocols on MEP amplitudes. a and $\mathbf{b}$ Plot the mean pre- and post-MEP amplitudes at all timepoints in all 6 stimulation conditions. $\mathbf{c}$ and d Simplify the data by plotting the mean data at all timepoints post-TBS together with the mean pre-TBS values. ANOVA conducted on the data in $\mathbf{a}$, b revealed no effect for the time course. e Provides a summary of the results expressed as normalised mean MEP values. Data are presented as means \pm SEM. For detailed analysis see Table 1
tDCS + iTBS
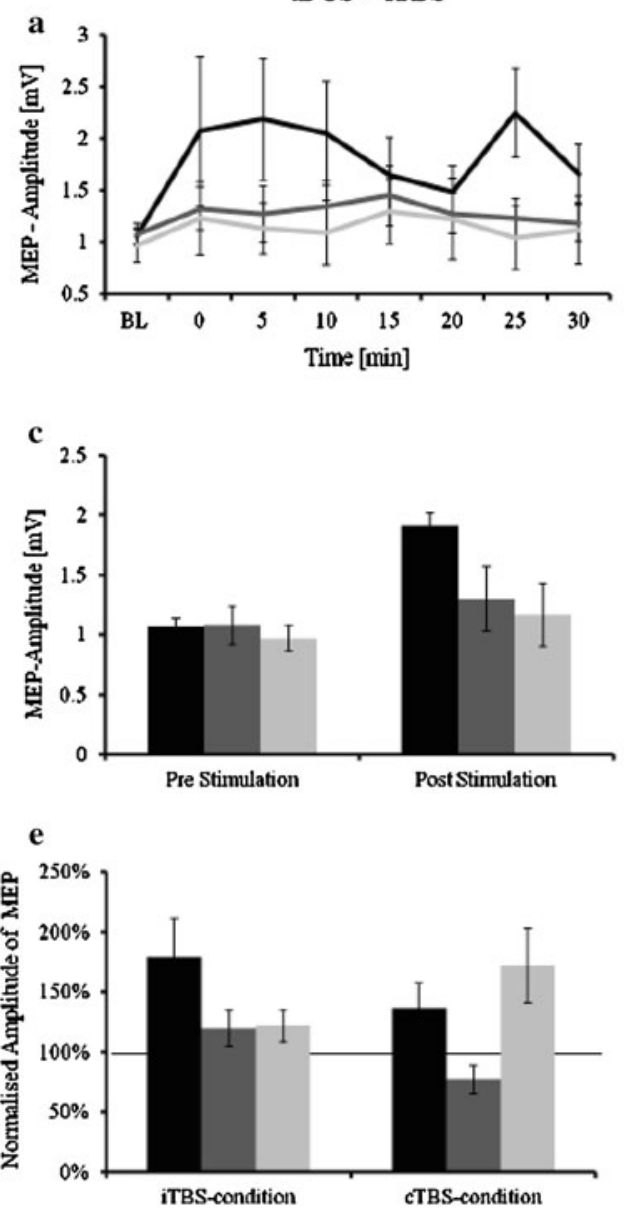

tDCS + cTBS
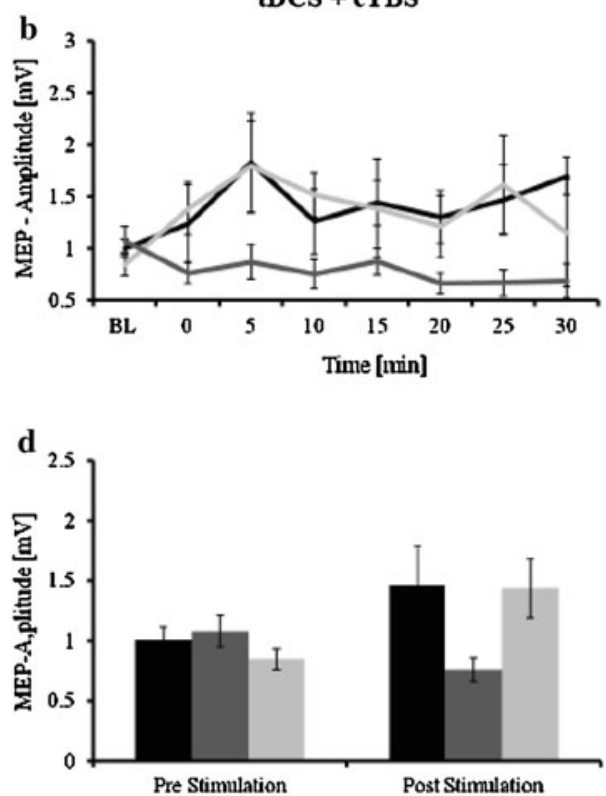

Cathodal tDCS

Sham tDCS

Anodal tDCS 
Table 1 Results of the ANOVAs (MEP size) for the different experiments

\begin{tabular}{llll}
\hline Experiments & Df, Error & $F$ value & $P$ value \\
\hline All conditions (Timecourse) & & & \\
Condition $(n=6)$ & $2.1,16.7$ & 3.789 & $0.043^{*}$ \\
Timecourse & $3.6,28.6$ & 2.582 & 0.064 \\
Condition $\times$ Timecourse & $4.0,32.0$ & 0.992 & 0.486 \\
All conditions (pre/post) & & & \\
Condition $(n=6)$ & 5,40 & 2.789 & $0.030^{*}$ \\
Time (pre/post) & 1,8 & 2.979 & $0.039^{*}$ \\
Condition $\times$ Time & $1.7,17.3$ & 4.182 & $0.031^{*}$ \\
\hline
\end{tabular}

$* P<0.05$

condition $(t=2.983, d f=8, P=0.020)$ and the anodaltDCS + iTBS condition $(P=0.080$, trend level $)$. MEP amplitudes in the sham-tDCS + iTBS and anodal-tDCS + iTBS condition did not differ after stimulation $(P=0.562)$. Paired $t$ tests showed that compared with baseline, MEPs were facilitated in the cathodal-tDCS + iTBS condition $(t=2.435, d f=8, P=0.041)$, but not in the two other conditions $(t=1.489, d f=8, P=0.175$ for
sham-tDCS + iTBS condition and $t=1.426, d f=8$, $P=0.192$ for anodal-tDCS + iTBS condition).

For cTBS, post hoc $t$ tests showed that the post-TBS MEPs were larger after either anodal-tDCS + cTBS or cathodal-tDCS + cTBS as compared to sham-tDCS + cTBS $(t=2.535, d f=8, P=0.035 ; t=3.217, d f=8, P=0.012)$. Moreover, MEPs in the anodal-tDCS + cTBS condition were significantly larger than at baseline before intervention $(t=2.534, d f=8, \quad P=0.035)$, and the cathodaltDCS + cTBS condition showed a numeric enlargement of MEP amplitude without reaching significance $(t=-1.807$, $d f=8, P=0.108)$. Sham-tDCS-cTBS reduced MEP amplitudes $(t=-2.180, d f=8, P=0.061$, trend). MEP amplitudes were significantly larger after sham-tDCS + iTBS compared with sham-tDCS + cTBS $(t=02.500, d f=8$, $P=0.037)$.

\section{Correlation analysis}

Simultaneous tDCS changed the after effect of TBS in three conditions significantly: cathodal-tDCS + iTBS, cathodal-tDCS + cTBS and anodal-tDCS + cTBS. In
Fig. 2 Results of the pairedpulse TMS measurements. Short-latency intracortical inhibition (SICI) and facilitation (ICF) were obtained for interstimulus intervals of 2-12 ms. Results are expressed as percentage of the test-MEP amplitude (normalised values). a Cathodal-tDCS + iTBS, b sham-tDCS + iTBS, c anodal-tDCS + iTBS, d cathodal-tDCS + iTBS, e sham-tDCS + iTBS, $\mathbf{f}$ anodaltDCS + iTBS. No significant effects for SICI or ICF were revealed by ANOVA (see text). Data are presented as means \pm SEM
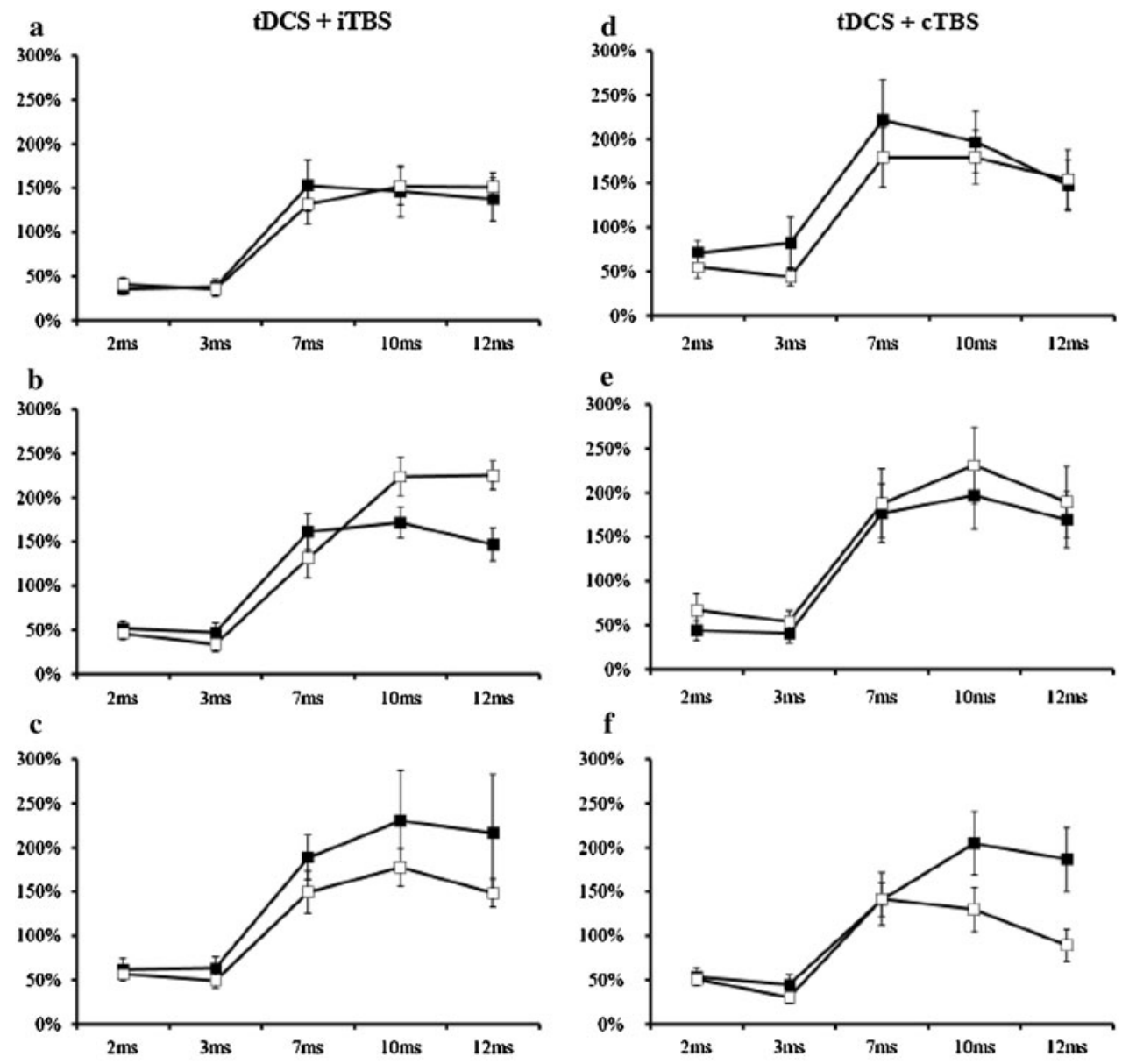

Pre Stimulation $\square$ Post Stimulation 


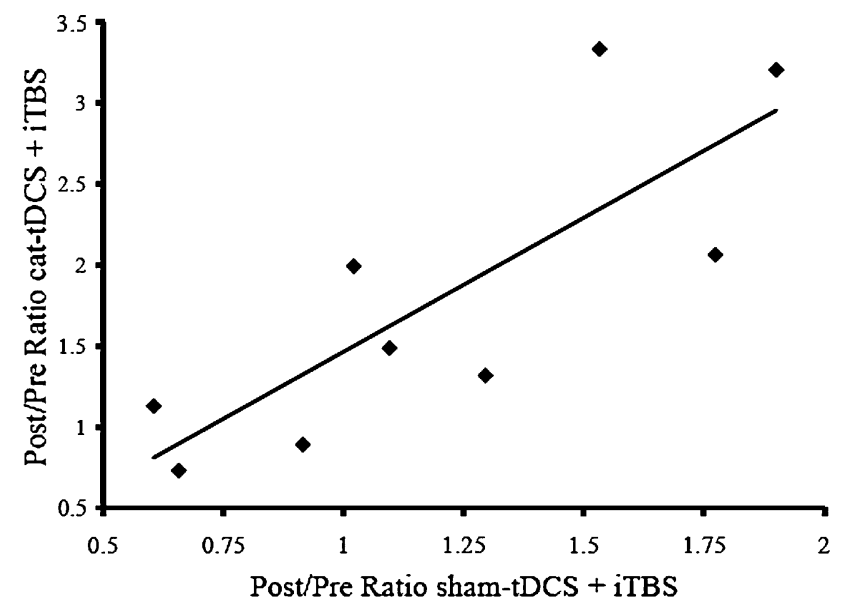

Fig. 3 Correlation $(P=0.008)$ between the post/pre-MEP ratios of cathodal-tDCS + iTBS ( $y$ axis) and sham-tDCS + iTBS ( $x$ axis). The slope is 1.66

these three cases, we tested whether the post/pre-MEP ratio with real tDCS was related to the ratio in the sham tDCS conditions. We found a significant positive correlation between the post/pre-MEP ratio of the cathodaltDCS + iTBS condition and the sham-tDCS + iTBS condition (p.c. $=0.808, P=0.008$, B-Coefficient $=1.66$, $R=0.808$ see Fig. 3). Neither of the two other post/precorrelations was significant.

\section{Effects on motor thresholds}

Resting and active motor thresholds were not altered by the interventions. RM-ANOVA for RMT showed no significant main effects of "condition" $(F(5,40)=0.908, P=0.485)$ or "time" $(F(1,8)=4.558, P=0.065)$ and no significant "condition $\times$ time" interaction $(F(1.6,13.3)=1.423, P=$ 0.271). For AMT, RM-ANOVA displayed no significant effects of "condition" $(F(5,40)=1.999, P=0.100)$ or "time" $(F(1,8)=0.151, P=0.708)$ and no significant "condition $\times$ time" interaction $(F(1.2,9.7)=1.152, P=0.349)$. For details see Table 2.
Effects on intracortical inhibition and facilitation

Since SICI and ICF are thought to be due to different mechanisms (Ziemann et al. 1996b), we analysed the data separately for ISIs of $2 / 3 \mathrm{~ms}$ (SICI), $7 \mathrm{~ms}$ (intermediate) and $10 / 12 \mathrm{~ms}$ (ICF). None of the interventions had any effect on the measures, apart from a significant effect of "ISI" in the ICF paradigm. This was due to the fact that ICF was larger at ISI $=12 \mathrm{~ms}$ than at ISI $=10 \mathrm{~ms}$ (see Table 3 ).

\section{Discussion}

The present results show that the LTP-/LTD-like after effects of iTBS/cTBS on motor cortex excitability are significantly modulated by simultaneous tDCS. Cathodal tDCS increases the facilitatory effect of iTBS and abolishes the effect of cTBS; anodal tDCS has no significant influence on the response to iTBS, but suppresses and even reverses effect of cTBS. None of the protocols influenced SICI/ICF or threshold measures.

The interpretation of these effects is complex because tDCS has three potential consequences that could interact with the LTP/LTD-like changes caused by TBS. Thus, tDCS can (1) bias the membrane potential of cortical neurones and thereby change their response to theta-burst protocols (Artola et al. 1990); (2) change the ongoing level of activity in cortical networks, with anodal stimulation increasing basal activity and cathodal stimulation reducing it (Bindman et al. 1964) which could interact with TBS according to "homoeostatic" rules; (3) if applied for longer than 3 min (Nitsche and Paulus 2000, 2001), tDCS causes long-term LTP- and LTD-like changes in synaptic connections that could change the response to TBS.

Interestingly, each of these three possible explanations has been used to account for the results of previous studies of tDCS and TMS. In their initial report, Nitsche \& Paulus interacted tDCS and single-pulse TMS. They found that MEPs were enhanced when applied during a short period of anodal tDCS; in contrast, MEPs were reduced by simultaneous

Table 2 Mean values of resting motor thresholds (RMT), active motor thresholds (AMT, active motor thresholds obtained with a biphasic stimulator $\left(\mathrm{AMT}_{\mathrm{Biphasic}}\right)$, TMS intensity to evoke a MEP with a $1 \mathrm{mV}$ peak-to-peak size $(\mathrm{S} 1 \mathrm{mV})$

\begin{tabular}{|c|c|c|c|c|c|c|}
\hline Intervention & RMT Pre & RMT Post & AMT Pre & AMT Post & $\mathrm{AMT}_{\text {biphasic }}$ & SI1 mV \\
\hline Cathodal - tDCS + iTBS & $40.7 \pm 1.3$ & $40.4 \pm 1.2$ & $31.8 \pm 1.3$ & $31.0 \pm 1.2$ & $54.1 \pm 1.4$ & $47.8 \pm 1.4$ \\
\hline Anodal - tDCS + iTBS & $42.7 \pm 0.9$ & $42.2 \pm 0.8$ & $31.4 \pm 1.1$ & $30.1 \pm 1.5$ & $55.0 \pm 2.5$ & $48.1 \pm 1.3$ \\
\hline Sham - tDCS + iTBS & $41.1 \pm 1.1$ & $40.8 \pm 1.2$ & $31.3 \pm 1.6$ & $31.3 \pm 1.5$ & $55.6 \pm 2.2$ & $47.6 \pm 1.4$ \\
\hline Cathodal - tDCS + cTBS & $41.2 \pm 1.9$ & $40.1 \pm 1.7$ & $32.3 \pm 1.5$ & $29.8 \pm 1.6$ & $54.6 \pm 2.6$ & $46.7 \pm 1.9$ \\
\hline Anodal - tDCS + cTBS & $41.3 \pm 1.5$ & $40.3 \pm 1.4$ & $31.4 \pm 1.1$ & $30.1 \pm 1.1$ & $54.1 \pm 2.0$ & $48.3 \pm 1.9$ \\
\hline Sham - tDCS + cTBS & $42.1 \pm 1.7$ & $42.7 \pm 1.8$ & $32.0 \pm 1.9$ & $31.7 \pm 1.7$ & $55.1 \pm 1.9$ & $47.7 \pm 2.4$ \\
\hline
\end{tabular}

Thresholds were measured before and after the stimulation. Data are presented as means \pm SEM 
Table 3 Results of the ANOVAs (Paired-pulse TMS) for the different experiments

\begin{tabular}{llll}
\hline Experiments & $D f$, Error & $F$ value & $P$ value \\
\hline SICI & & & \\
Condition & 5,40 & 1.943 & 0.848 \\
Time (pre/post) & 1,8 & 1.152 & 0.314 \\
ISI $(2,3)$ & 1,8 & 0.804 & 0.396 \\
Condition $\times$ Time & 5,40 & 1.842 & 0.127 \\
Condition $\times$ ISI & 5,40 & 0.397 & 0.848 \\
ISI $\times$ Time & 1,8 & 4.374 & 0.070 \\
Condition $\times$ Time $\times$ ISI & 5,40 & 0.135 & 0.983 \\
ICF & & & \\
Condition & 5,40 & 1.155 & 0.384 \\
Time $($ pre/post) & 1,8 & 0.422 & 0.543 \\
ISI $(10,12)$ & 1,8 & 2.913 & $0.019 *$ \\
Condition $\times$ Time & $1.5,12.2$ & 1.754 & 0.145 \\
Condition $\times$ ISI & $3.2,25.7$ & 1.001 & 0.430 \\
ISI $\times$ Time & 1,8 & 0.001 & 0.145 \\
Condition $\times$ Time $\times$ ISI & 5,40 & 0.406 & 0.841 \\
Intermediate ISI & & & \\
Condition & $2.1,17.1$ & 1.539 & 0.243 \\
Time $($ pre $/$ post $)$ & 1,8 & 1.111 & 0.323 \\
Condition $\times$ Time & $2.6,21.1$ & 0.355 & 0.761 \\
\hline & & &
\end{tabular}

$* P<0.05$

cathodal tDCS (Nitsche and Paulus 2001). This finding was replicated in a more recent study (Nitsche et al. 2005) which showed that a short period of tDCS, which elicits no after effects, had no effect on resting or active motor thresholds nor on the amount of paired-pulse inhibition and facilitation (Kujirai et al. 1993), when measured during tDCS. These changes were interpreted in terms of the tDCS effect on membrane potential. It was suggested that short-lasting tDCS deor hyperpolarises the cell bodies of corticospinal pyramidal neurones, making the neurons more/less likely to respond with an action potential to a given excitatory input. The lack of effect on SICI during short-lasting tDCS appeared to indicate that $\mathrm{GABA}_{\mathrm{A}}$-interneurons were unaffected by tDCS. This is in line with the observation that application of Lorazepam, a $\mathrm{GABA}_{\mathrm{A}}$-receptor agonist, had no effect on the response to short-lasting tDCS (Nitsche et al. 2004).

The only other experiment investigating simultaneous tDCS and TMS has involved a more complex combination of long-lasting tDCS with the facilitatory paired associative stimulation protocol (PAS) (Nitsche et al. 2007), which is thought to induce a form of NMDA-dependent spike-timing-dependent plasticity (STDP) within motor cortex. Simultaneous application of cathodal-tDCS prolonged the LTP-like effects of PAS, whereas anodal-tDCS turned the LTP-like effects to inhibition. The authors interpreted these changes in terms of tDCS effects on ongoing cortical activ- ity. They used a homoeostatic argument to suggest that cathodal tDCS reduces background activity (Bindman et al. 1964), which will favour the induction of LTP, whereas enhanced background activity, generated by anodal tDCS, will turn LTP into LTD (Nitsche et al. 2007).

Several other studies have examined what happens when longer periods of tDCS ( $>5 \mathrm{~min}$ ) are followed by another plasticity protocol. However, consecutive application of plasticity protocols in awake humans leads to a variety of complex interactions that may or may not follow simple homoeostatic logic (Ridding and Ziemann 2010). This could be due to the fact that long periods of tDCS not only change the prior history of ongoing activity in the network, but can themselves produce LTD- and LTP-like after effects (see Introduction) that may have separate interactions with the plasticity protocol.

As in these previous studies, we can only speculate on which type of interaction might be most likely to account for our present results. We will consider them in turn.

\section{Homoeostatic explanation}

The combination of cathodal tDCS with iTBS resulted in an enhanced LTP-like plasticity and cathodal tDCS abolished LTD-like plasticity after cTBS. Both of these effects could result from "homoeostatic" interactions as proposed in the tDCS/PAS study by Nitsche et al. (2007). Cathodal tDCS might reduce ongoing activity and enhance LTP-like effects of iTBS whilst reducing LTD-like effects of cTBS. However, the effects of anodal tDCS are less compatible with this explanation. Anodal tDCS had no significant effect on the response to iTBS, although it can be argued that since the effect of iTBS-sham was relatively small, changes produced by anodal tDCS may not have been detectable in the present experiments. A more important exception to the homoeostatic explanation was the fact that anodal tDCS changed the response produced by cTBS from inhibition to facilitation. This is contrary to the expectations of homoeostatic rules in which it should promote LTD-like effects.

\section{Membrane polarising effect of tDCS}

Artola et al. (1990) found that in slice preparations, depolarisation of the postsynaptic membrane favoured production of LTP, whereas hyperpolarisation initially favoured LTD; if hyperpolarisation was too strong, then neither LTP nor LTD could be produced. First, consider the effects of cathodal tDCS which were well-fitted by the homoeostatic explanation above. Cathodal tDCS hyperpolarises the cell bodies of pyramidal neurones in the cortex whilst simultaneously depolarising their distal dendrites (Creutzfeldt 
et al. 1962). If the synapses activated by TBS are onto distal dendrites, then cathodal tDCS should favour LTP-like changes. The result would be the same as the homoeostatic explanation above: cathodal tDCS would increase the response to iTBS and reduce the response to cTBS.

In contrast, anodal tDCS depolarises cell bodies of pyramidal neurones whilst hyperpolarising their dendrites: hyperpolarisation of the postsynaptic membrane initially favours induction of LTD-like effects, but then at greater levels of hyperpolarisation, no LTD-like effects can be produced. If anodal tDCS had this latter effect, then the response to cTBS would be abolished. However, anodal tDCS did not simply suppress the response to cTBS, it changed its polarity from depression to facilitation. One explanation for this relates to the basic mechanism of inhibition following cTBS600. Gentner and colleagues noted that short periods of cTBS (300 stimuli) can produce facilitation rather than inhibition, and they suggested that longer periods of cTBS600 yielded inhibition because of excess $\mathrm{Ca}^{2+}$-entry into the neurones provoked by continuous stimulation (Gentner et al. 2008). Hyperpolarisation of the dendrites by anodal tDCS might lower excitability and reduce the $\mathrm{Ca}^{2+}$-overflow induced by cTBS. A reduction of $\mathrm{Ca}^{2+}$-overflow after cTBS 600 might lead to the facilitatory effect seen after cTBS 300 .

\section{Interactions with LTP/LTD-like effects of tDCS and TBS}

In our study, tDCS was given for a very short period of time ( $<3.5 \mathrm{~min}$ when simultaneous with iTBS; $<1 \mathrm{~min}$ when simultaneous with cTBS). When applied alone, tDCS of this duration has no lasting LTP- and LTD-like effects that might interact with those of TBS (Nitsche and Paulus 2001). Thus, any effect that tDCS have on the response to TBS is likely to occur because it changes the way neurones react when TBS is applied, rather than to some complex interaction between their respective LTP/LTD-like after effects. However, it is not possible to rule out subthreshold persisting changes from even short duration periods of tDCS which could potentially interact with the lasting effects of TBS. This could perhaps be addressed in future experiments in which sequential applications of very short periods of tDCS and TBS could be explored to test for time-dependent interactions between protocols.

\section{Effects on SICI/ICF and motor thresholds}

None of the protocols had any effect on SICI/ICF or thresholds. Since neither AMT nor RMT are affected individually by tDCS or TBS, it is not surprising that the combined intervention had no effect on these parameters (Huang et al.
2005; Nitsche and Paulus 2001). Both SICI and ICF have been reported to change after separate application of both TBS and long periods of tDCS. However, a very short period of tDCS as used here $(<3 \mathrm{~min})$ is not known to have any lasting effects on either circuit. At first sight, our SICIF/ ICF findings seem contrary to the original observations of Huang et al. (2005) who reported that SICI was suppressed after cTBS and enhanced after iTBS; in addition, ICF was reduced after iTBS. However, these effects were maximum about 10 min after stimulation. Because of the need to evaluate changes in MEP as well as thresholds, our measures of SICI/ICF were taken at $5 \mathrm{~min}$ and therefore, we may have missed these baseline effects. In addition, our strong conditioning pulse ( $80 \%$ RMT) may be a methodological limitation and may result in floor or ceiling effects in our paired-pulse experiments. Finally, it is possible that the SICI and ICF networks are not involved in the generation of the observed effects.

\section{Limitations}

In contrast to the original TBS publication (Huang et al. 2005), we found only a numeric enhancement of MEPs after iTBS (20\%, compared with up to $100 \%$ in the original publication) and only a moderate inhibition of MEPs after cTBS (25\%, up to $50 \%$ in the original publication). Nevertheless, MEPs were significantly larger following sham-tDCS + iTBS compared with sham-tDCS + cTBS. Our data are more similar to those of Todd et al. (2009) who reported no significant effect neither for iTBS nor for cTBS and who postulated that this was related to the large inter-subject variability in response to TBS. Finally, it should be considered that we performed TBS through a tDCS electrode (thickness $5 \mathrm{~mm}$ ). Though correcting for this coil-to-scalp-distance by increasing the stimulus intensity, a change in the distribution of the electrical field may contribute to the reduced TBS effects. One study found modulations in cortical network activation related to the distance between coil and skull (Cukic et al. 2009), and it is possible that this could have confounded our findings.

\section{Conclusions}

In summary, the present study indicates that tDCS has the potential to interact with motor cortex plasticity generated by TBS. Our results give slightly stronger support to the membrane polarisation hypothesis than the "homoeostatic" interaction, but we would not dismiss more complex accounts involving mixtures of several effects. With regard to the clinical application of non-invasive brain 
stimulations, it may be important to combine stimulation protocols to get optimal stimulation effects. Our results show that concurrent cathodal tDCS can stabilise the effect of iTBS and turn the usual cTBS effect into LTP-like plasticity which may be useful in future clinical applications. This might have further importance for diseases with ongoing activity modifications of cortical areas (e.g. schizophrenia, Tourette's diseases) which are treated with TBS.

Acknowledgments This work was supported by EU FP7 Collaborative Project (223524: PLASTICISE). Alkomiet Hasan is supported by the Deutsche Forschungsgemeinschaft (DFG grant HA 6091/1-1). Masashi Hamada is supported by the Japan Society for the Promotion of Science Postdoctoral Fellowships for Research Abroad.

Conflict of interest The authors have no potential conflict of interests related to the subject of this report.

Open Access This article is distributed under the terms of the Creative Commons Attribution Noncommercial License which permits any noncommercial use, distribution, and reproduction in any medium, provided the original author(s) and source are credited.

\section{References}

Artola A, Singer W (1993) Long-term depression of excitatory synaptic transmission and its relationship to long-term potentiation. Trends Neurosci 16:480-487

Artola A, Brocher S, Singer W (1990) Different voltage-dependent thresholds for inducing long-term depression and long-term potentiation in slices of rat visual cortex. Nature 347:69-72

Bindman LJ, Lippold OC, Redfearn JW (1964) The Action of Brief Polarizing Currents on the Cerebral Cortex of the Rat (1) during Current Flow and (2) in the Production of Long-Lasting afterEffects. J Physiol 172:369-382

Creutzfeldt OD, Fromm GH, Kapp H (1962) Influence of transcortical d-c currents on cortical neuronal activity. Exp Neurol 5:436-452

Cukic M, Kalauzi A, Ilic T, Miskovic M, Ljubisavljevic M (2009) The influence of coil-skull distance on transcranial magnetic stimulation motor-evoked responses. Exp Brain Res 192:53-60

Di Lazzaro V, Oliviero A, Profice P, Saturno E, Pilato F, Insola A, Mazzone P, Tonali P, Rothwell JC (1998) Comparison of descending volleys evoked by transcranial magnetic and electric stimulation in conscious humans. Electroencephalogr Clin Neurophysiol 109:397-401

Gentner R, Wankerl K, Reinsberger C, Zeller D, Classen J (2008) Depression of human corticospinal excitability induced by magnetic theta-burst stimulation: evidence of rapid polarity-reversing metaplasticity. Cereb Cortex 18:2046-2053

Huang YZ, Edwards MJ, Rounis E, Bhatia KP, Rothwell JC (2005) Theta burst stimulation of the human motor cortex. Neuron 45:201-206

Huang YZ, Rothwell JC, Chen RS, Lu CS, Chuang WL (2010) The theoretical model of theta burst form of repetitive transcranial magnetic stimulation. Clin Neurophysiol doi:10.10167j.clinph.2010.08.016

Kujirai T, Caramia MD, Rothwell JC, Day BL, Thompson PD, Ferbert A, Wroe S, Asselman P, Marsden CD (1993) Corticocortical inhibition in human motor cortex. J Physiol 471:501-519

Nitsche MA, Paulus W (2000) Excitability changes induced in the human motor cortex by weak transcranial direct current stimulation. J Physiol 527(Pt 3):633-639

Nitsche MA, Paulus W (2001) Sustained excitability elevations induced by transcranial DC motor cortex stimulation in humans. Neurology 57:1899-1901

Nitsche MA, Liebetanz D, Schlitterlau A, Henschke U, Fricke K, Frommann K, Lang N, Henning S, Paulus W, Tergau F (2004) GABAergic modulation of DC stimulation-induced motor cortex excitability shifts in humans. Eur J Neurosci 19:2720-2726

Nitsche MA, Seeber A, Frommann K, Klein CC, Rochford C, Nitsche MS, Fricke K, Liebetanz D, Lang N, Antal A, Paulus W, Tergau F (2005) Modulating parameters of excitability during and after transcranial direct current stimulation of the human motor cortex. J Physiol 568:291-303

Nitsche MA, Roth A, Kuo MF, Fischer AK, Liebetanz D, Lang N, Tergau F, Paulus W (2007) Timing-dependent modulation of associative plasticity by general network excitability in the human motor cortex. J Neurosci 27:3807-3812

Nitsche MA, Cohen LG, Wassermann EM, Priori A, Lang N, Antal A, Paulus W, Hummel F, Boggio PS, Fregni F, Pascual-Leone A (2008) Transcranial direct current stimulation: State of the art 2008. Brain Stimul 1:206-223

Oldfield RC (1971) The assessment and analysis of handedness: the Edinburgh inventory. Neuropsychologia 9:97-113

Ridding MC, Ziemann U (2010) Determinants of the induction of cortical plasticity by non-invasive brain stimulation in healthy subjects. J Physiol 588:2291-2304

Ridding MC, Taylor JL, Rothwell JC (1995) The effect of voluntary contraction on cortico-cortical inhibition in human motor cortex. J Physiol 487(Pt 2):541-548

Rothwell JC, Hallett M, Berardelli A, Eisen A, Rossini P, Paulus W (1999) Magnetic stimulation: motor evoked potentials. The International Federation of Clinical Neurophysiology. Electroencephalogr Clin Neurophysiol Suppl 52:97-103

Talelli P, Cheeran BJ, Teo JT, Rothwell JC (2007) Pattern-specific role of the current orientation used to deliver Theta Burst Stimulation. Clin Neurophysiol 118:1815-1823

Todd G, Flavel SC, Ridding MC (2009) Priming theta-burst repetitive transcranial magnetic stimulation with low- and high-frequency stimulation. Exp Brain Res 195:307-315

Ziemann U, Siebner HR (2008) Modifying motor learning through gating and homeostatic metaplasticity. Brain Stimul 1:60-66

Ziemann U, Lonnecker S, Steinhoff BJ, Paulus W (1996a) Effects of antiepileptic drugs on motor cortex excitability in humans: a transcranial magnetic stimulation study. Ann Neurol 40:367-378

Ziemann U, Rothwell JC, Ridding MC (1996b) Interaction between intracortical inhibition and facilitation in human motor cortex. J Physiol 496(Pt 3):873-881

Ziemann U, Paulus W, Nitsche MA, Pascual-Leone A, Byblow WD, Berardelli A, Siebner HR, Classen J, Cohen LG, Rothwell JC (2008) Consensus: Motor cortex plasticity protocols. Brain Stimul 1:164-182 